\title{
Aperçu
}

\section{Prise en charge des traumatismes crâniens au Canada : évolution des tendances en matière de soins}

\author{
Deepa P. Rao, Ph. D.; Steven McFaull, M. Sc.; Wendy Thompson, M. Sc.; Gayatri C. Jayaraman, Ph. D.
}

Diffuser cet article sur Twitter

Résumé

Introduction : Malgré une plus grande sensibilisation aux traumatismes crâniens, nous disposons de peu de renseignements à l'échelle de la population sur les types de soins prodigués en lien avec les traumatismes crâniens au Canada.

Méthodologie : Nous avons examiné les données de l'Enquête sur la santé dans les collectivités canadiennes (années 2005, 2009 et 2014) portant sur les répondants de 12 ans et plus. Les variables sélectionnées en lien avec la prise en charge des traumatismes crâniens ont été l'accès à des soins dans les 48 heures suivant la blessure, le point de service, l'admission à l'hôpital et les soins de suivi.

Résultats : Nous avons constaté qu'un grand nombre de Canadiens avaient bénéficié de soins médicaux dans les 48 heures suivant leur blessure, sans changement dans cette proportion au fil du temps. Nous avons aussi observé un déclin significatif de la proportion de Canadiens ayant décidé de se rendre au service des urgences ( $p=0,03$, tous âges confondus) et une augmentation significative du nombre de jeunes ayant décidé de consulter un médecin $(p<0,01)$.

Conclusion : Les traumatismes crâniens sont un problème de santé important et en augmentation au Canada. Ces blessures semblent de plus en plus être traitées par des professionnels de la santé en dehors des hôpitaux, en particulier par des médecins de première ligne.

Mots-clés : traumatisme crânien, commotion cérébrale, surveillance

\section{Introduction}

Rowan Stringer est décédée des suites d'une commotion cérébrale après avoir reçu un coup à la tête en jouant au rugby pour son école secondaire. À la suite de son décès, une grande initiative visant à accroître la sensibilisation aux commotions cérébrales et à améliorer les traitements a abouti à la sanction royale de la Loi de 2016 sur le Comité consultatif de la Loi Rowan en 2016 ${ }^{1}$. De plus en plus de professionnels partout au Canada investissent maintenant dans la prévention et la lutte contre les commotions cérébrales et les autres traumatismes crâniens. Selon une étude récente, l'incidence des traumatismes crâniens est en hausse au Canada : la variation annuelle en pourcentage (VAP) des traumatismes crâniens chez les personnes ayant déclaré une blessure sérieuse au cours des 12 derniers mois a été de 9,6\% (IC à $95 \%: 8,2$ à 11,0$)^{2}$.

La difficulté à diagnostiquer de façon précise et opportune les traumatismes crâniens sème des doutes sur cette prétendue " épidémie invisible " $^{3}$. Que leur état ait été diagnostiqué ou non, une partie des victimes de traumatisme crânien présente un risque de syndrome post commotionnel persistant (c. à d. persistance des symptômes sur plusieurs semaines ou mois ${ }^{4,5}$ ). Les jeunes athlètes présentent un risque
Points saillants

- Environ 80 \% des Canadiens ayant déclaré un traumatisme crânien ont bénéficié de soins médicaux dans les 48 heures suivant leur blessure.

- L'examen des tendances des 10 dernières années montre un déclin significatif de la proportion de jeunes ayant déclaré s'être rendus à un service des urgences à la suite d'un traumatisme crânien.

- Selon les données actuelles, environ 1 Canadien sur 10 (à interpréter avec prudence) ayant déclaré avoir subi un traumatisme crânien dit s'être rendu à un cabinet médical à la suite de sa blessure.

(quoique faible) de syndrome du second impact, c'est à dire de commotion cérébrale survenant avant la guérison d'une commotion antérieure ${ }^{6}$. Il est important de détecter les traumatismes crâniens, étant donné qu'une erreur de diagnostic ou de traitement peut entraîner une invalidité voire la mort ${ }^{7,8}$. En ce qui a trait à l'accès aux soins médicaux, selon les plus récentes lignes directrices canadiennes et internationales concernant les commotions cérébrales liées à la pratique d'un sport, si on soupçonne qu'une personne a subi un traumatisme crânien, on doit la retirer du jeu et la faire évaluer par un médecin ou par un professionnel de la santé qualifié9,10. Le lieu où se rendent les personnes pour obtenir des soins semble être en train de changer : une étude récente a révélé une transition des services des urgences vers les fournisseurs de soins primaires et spécialisés ${ }^{11,12}$. 
Cette analyse descriptive a pour objectif de fournir des estimations à l'échelle de la population concernant la prise en charge des traumatismes crâniens : les victimes obtiennent elles des soins médicaux dans les 48 heures suivant leur blessure? Où se rendent elles pour être traitées? Sont elles hospitalisées ou non? Reçoivent elles des soins de suivi? Compte tenu du peu de renseignements disponibles à l'échelle nationale sur les traumatismes crâniens au Canada, notre étude a aussi pour objectif secondaire de se servir des questions utilisées dans les enquêtes nationales pour faire ressortir les tendances sur le plan de la prise en charge des traumatismes crâniens au fil du temps.

\section{Méthodologie}

\section{Sources de données}

L'Enquête sur la santé dans les collectivités canadiennes $^{13}$ (ESCC) est une enquête transversale sur la santé des Canadiens de 12 ans et plus excluant les personnes vivant dans des centres d'accueil, dans des établissements de soins de longue durée ou dans des réserves, les membres à temps plein des Forces armées canadiennes et les résidents civils des bases militaires. L'enquête a été conçue pour fournir des estimations à l'échelle nationale et provinciale ainsi qu'à l'échelle des 110 régions sanitaires du Canada. Nous avons utilisé des données autodéclarées provenant de l'ESCC (années 2005, 2009 et 2014) pour mettre en évidence les caractéristiques associées aux traumatismes crâniens au sein de la population canadienne de 12 ans et plus (jeunes : 12 à 17 ans; adultes : 18 ans et plus).

\section{Variables clés}

Nous avons considéré comme victime de traumatisme crânien toute personne ayant déclaré une blessure "survenue durant les 12 derniers mois [et] suffisamment grave pour limiter les activités normales le lendemain de la blessure » et ayant identifié cette blessure comme un traumatisme crânien. Les variables liées à la prise en charge des traumatismes crâniens visées par notre analyse ont été examinées uniquement chez les personnes ayant autodéclaré un traumatisme crânien et sont les suivantes : i) accès à des soins dans les 48 heures suivant la blessure, ii) point de service, iii) admission à l'hôpital pour la nuit et iv) prestation de soins de suivi. Ces variables, sélectionnées en fonction de la disponibilité des questions de l'enquête sur les soins obtenus, ne correspondent à aucune recommandation en matière de prise en charge des traumatismes crâniens.

La variable " accès à des soins dans les 48 heures suivant la blessure " a été définie à partir de la question correspondante de l'enquête visant à recueillir des renseignements sur le temps nécessaire pour accéder à des soins : " [Avez vous] reçu des soins médicaux prodigués par un professionnel de la santé dans les 48 heures suivant la blessure? » Les participants ayant répondu par l'affirmative ont ensuite eu à préciser où ils étaient allés pour obtenir des soins, ce qui a permis de déterminer le «point de service ", qui pouvait être un service des urgences comme un autre lieu (service de consultation externe, cabinet de chiropraticien, centre de santé communautaire, service de consultation externe d'un hôpital, cabinet médical, lieu de survenue du traumatisme crânien). Dans le cas des participants ayant déclaré avoir reçu un traitement dans les 48 heures, on a déterminé s'il y avait eu " admission à l'hôpital à la suite de la blessure » à l'aide de la question de l'enquête : " Avez-vous été admis à l'hôpital pour la nuit du fait de cette blessure? » Enfin, les renseignements concernant les soins de suivi ont été saisis au moment de la collecte de données à l'aide de la question : "Présentement, êtes vous suivi par un professionnel de la santé en raison de cette blessure? »

\section{Analyses statistiques}

Nous avons réalisé des analyses descriptives à l'aide du logiciel SAS Enterprise Guide, version 5.1 (Cary, Caroline du Nord, États-Unis). Nous avons pondéré les estimations des incidences et des proportions pour tenir compte de la population des ménages canadiens et nous avons calculé des intervalles de confiance à $95 \%$ à l'aide de méthodes de rééchantillonnage " bootstrap ». Nous avons utilisé des modèles logistiques généralisés pour estimer la variation annuelle en pourcentage (VAP) et le seuil de signification a été établi à $p<0,05$.

\section{Résultats}

En 2014, approximativement 4 personnes sur 5 ayant déclaré un traumatisme crânien ont obtenu des soins médicaux dans les 48 heures suivant leur blessure (tableau 1). Bien que la majorité d'entre elles se soient rendues à un service des urgences, une analyse au fil du temps indique que ce choix de point de service diminue fortement chez les jeunes (tableau 1) (VAP chez les jeunes : $-3,1 \%$, IC à 95 : $-3,8$ à $-2,4$, $p<0,001$; VAP chez les adultes : $-2,6 \%$, IC à $95 \%:-5,4$ à $0,3, p=0,08$; VAP tous âges confondus : $-2,7 \%$, IC à $95 \%$ : $-5,1$ à $-0,2, p=0,03)$. Si les estimations de 2005 et de 2009 sont trop peu nombreuses pour que des conclusions puissent en être dégagées, celles de 2014 permettent de constater qu'environ 1 personne sur 10 ayant subi un traumatisme crânien se rend maintenant à un cabinet médical $(11,5 \%$, IC à $95 \%: 5,2$ à 17,8 , à interpréter avec prudence). L'examen des tendances au fil du temps révèle une tendance significative à la hausse de cette proportion à se rendre à un cabinet médical surtout chez les jeunes (VAP chez les jeunes : 0,3\%, IC à $95 \%: 0,2$ à $0,3, p<0,001$; VAP chez les adultes : 0,06 \%, IC à $95 \%$ : $-0,07$ à 0,2 , $p=0,34$; VAP tous âges confondus : $0,1 \%$, IC à $95 \%: 0,02$ à $0,2, p=0,02)$.

Les données de 2005 montrent que 21,9 \% (IC à $95 \%: 12,7$ à 31,2, à interpréter avec prudence) des Canadiens ayant déclaré un traumatisme crânien ont été hospitalisés pour la nuit, contre 13,4\% (IC à $95 \%: 5,5$ à 21,3, à interpréter avec prudence) en 2014. Les personnes hospitalisées ont été plus nombreuses que les personnes non hospitalisées à déclarer qu'elles faisaient l'objet d'un suivi par un professionnel de la santé au moment de la collecte des données (figure 1). Quels que soient les antécédents d'hospitalisation, les données les plus récentes montrent que la majorité des personnes ayant déclaré un traumatisme crânien dans l'année ne faisaient pas l'objet d'un suivi par un professionnel de la santé au moment de leur participation à l'enquête (jeunes : 86,8 \%, IC à $95 \%$ : 77,8 à 95,9; adultes : 69,3\%, IC à $95 \%$ : 57,6 à 80,9 ; participants tous âges confondus : 73,5\%, IC à $95 \%: 64,2$ à 82,7).

\section{Analyse}

La hausse de fréquence des traumatismes crâniens tant au Canada ${ }^{2}$ qu'aux États Unis $^{14}$ soulève une question : où se rendent les Canadiens pour obtenir des soins en lien avec leur traumatisme crânien et de quels soins de suivi bénéficient-ils? En nous intéressant à ces questions, nous avons constaté des changements importants dans les lieux où les Canadiens décident de se rendre pour obtenir des soins : 
TABLEAU 1

Évolution sur dix ans des soins prodigués après un traumatisme crânien au sein de la population canadienne (ESCC, 2005, 2009 et 2014, données autodéclarées)

\begin{tabular}{|c|c|c|c|c|c|c|c|c|c|c|c|c|}
\hline & & \multicolumn{3}{|c|}{2005} & \multicolumn{3}{|c|}{2009} & \multicolumn{3}{|c|}{2014} & \multicolumn{2}{|c|}{$\begin{array}{l}\text { Variation annuelle } \\
\text { en pourcentage }\end{array}$} \\
\hline & & Effectifs & $\begin{array}{c}\text { Incidence } \\
(\%)\end{array}$ & IC à 95 \% & Effectifs & $\begin{array}{c}\text { Incidence } \\
(\%)\end{array}$ & IC à 95 \% & Effectifs & $\begin{array}{c}\text { Incidence } \\
(\%)\end{array}$ & IC à 95 \% & $\%$ & IC à 95 \% \\
\hline $\begin{array}{l}\text { Ont obtenu } \\
\text { des soins dans } \\
\text { les } 48 \text { heures } \\
\text { suivant la } \\
\text { blessure }\end{array}$ & $\begin{array}{l}\text { Tous } \\
\text { répondants } \\
\text { confondus }\end{array}$ & 38214 & 72,3 & 65,5 à 79,1 & 68525 & 83,8 & 75,3 à 92,3 & 123478 & 81,0 & 73,5 à 88,5 & $1,9 \%$ & $-1,9$ à 5,7 \\
\hline \multirow{3}{*}{$\begin{array}{c}\text { Ont été } \\
\text { traités dans } \\
\text { un service des } \\
\text { urgences }\end{array}$} & $\begin{array}{l}\text { Tous } \\
\text { répondants } \\
\text { confondus }\end{array}$ & 30879 & 80,8 & 73,2 à 88,4 & 45452 & 66,3 & 52,0 à 80,7 & 79037 & 64,0 & 53,1 à 74,9 & $-2,7 \%$ & $-5,1$ à $-0,2$ \\
\hline & Jeunes & 9396 & 81,0 & 68,3 à 93,7 & 9158 & 69,8 & 49,9 à 89,6 & 16878 & 61,5 & 45,4 à 77,6 & $-3,1 \%$ & $-3,8$ à $-2,4$ \\
\hline & Adultes & 21483 & 80,7 & 71,0 à 90,4 & 36295 & 65,5 & 48,5 à 82,5 & 62159 & 64,7 & 51,1 à 78,3 & $-2,6 \%$ & $-5,4$ à 0,3 \\
\hline
\end{tabular}

Source : ESCC (2005, 2009 et 2014).

Abréviations : ESCC, Enquête sur la santé dans les collectivités canadiennes; IC, intervalle de confiance.

Remarques : La catégorie des jeunes correspond aux répondants de 12 à 17 ans et la catégorie des adultes correspond aux répondants de 18 ans et plus.

Les estimations des traumatismes crâniens ont pour dénominateur l'ensemble des Canadiens ayant déclaré une blessure.

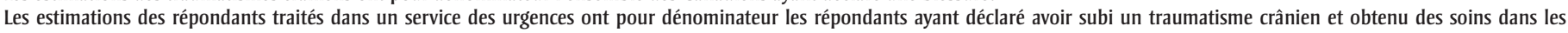
48 heures suivant la blessure.

Les estimations de la variation annuelle en pourcentage ont été calculées à l'aide de modèles logistiques généralisés.

les cabinets de médecin sont devenus un point de service important et de plus en plus courant et les jeunes sont de moins en moins nombreux à se rendre à un service des urgences. Nos constatations sont similaires à celles d'une étude récente sur la

\section{FIGURE 1}

Estimations de la fréquence des soins de suivi et des hospitalisations chez les Canadiens ayant subi un traumatisme crânien (ESCC, 2014, données autodéclarées)

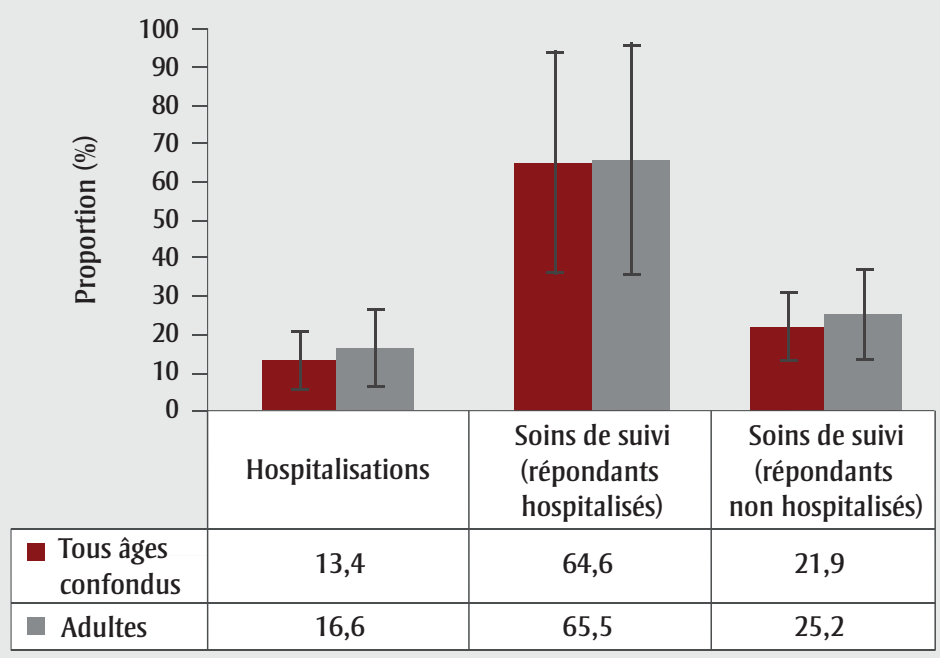

Source : ESCC, 2014.

Abréviation : ESCC, Enquête sur la santé dans les collectivités canadiennes.

Remarques : Les estimations concernant les jeunes étaient trop instables pour être fournies. Les estimations concernant les adultes concernent les personnes de 18 ans et plus.

Les estimations des traumatismes crâniens ont pour dénominateur l'ensemble des Canadiens ayant déclaré une blessure. Les estimations des soins de suivi (répondants hospitalisés) renvoient aux répondants ayant déclaré qu'ils recevaient des soins de suivi, qu'ils avaient été traités dans les 48 heures suivant leur blessure et qu'ils avaient été hospitalisés.

Les estimations des soins de suivi (répondants non hospitalisés) renvoient aux répondants ayant déclaré qu'ils recevaient des soins de suivi, qu'ils avaient été traités dans les 48 heures suivant leur blessure et qu'ils n'avaient pas été hospitalisés. et l'accessibilité des médecins de première ligne pourraient jouer un rôle. Nous n’avons remarqué aucun changement quant à la décision d'obtenir des conseils en matière de santé dans les 24 heures suivant un traumatisme crânien. La récente augmentation des traumatismes crâniens a été attribuée à divers facteurs, dont l'amélioration de la saisie des données sur les traumatismes crâniens légers ${ }^{16,17}$. En revanche, selon une évaluation récente des consignes relatives au retour au jeu à la suite d'une commotion cérébrale ${ }^{18}$, et bien que les recherches dans ce secteur en soient encore à leurs débuts, rien n'indiquerait que ces consignes aient eu un effet sur le pronostic de traumatisme crânien ${ }^{19}$. D'autres études sur les facteurs associés à la hausse des traumatismes crâniens ainsi que sur le pronostic de traumatisme crânien devraient aider à mieux orienter les lignes directrices fondées sur des preuves concernant la prise en charge des traumatismes crâniens ${ }^{19}$.

\section{Points forts et limites}

Les enquêtes nationales fondées sur l'autodéclaration des données ne tiennent pas compte des cas fatals. L'autodéclaration présente un risque de biais attribuable aux répondants, car la validité des résultats peut être affectée par le rappel rétrospectif des blessures autodéclarées ${ }^{20}$. L'exclusion des cas fatals a de fait limité nos constatations aux cas non fatals. En ce qui 
concerne l'examen des soins de suivi, la formulation de la question a empêché de distinguer les cas où le répondant avait fini de recevoir des soins de suivi au moment de l'enquête de ceux où les soins de suivi n'avaient pas encore été dispensés. Cette question a néanmoins permis de faire ressortir des différences entre certains groupes clés, en particulier entre les répondants ayant été hospitalisés et les autres. Bien que notre étude comporte des limites sur le plan de la validité interne et externe, elle a permis de mieux saisir les cas diagnostiqués ailleurs que dans un service des urgences, ce qui constitue l'une de ces forces. De plus, comme la plupart des traumatismes crâniens ne sont pas fatals (seulement environ $3 \%$ le sont ${ }^{14}$ ), nos constatations sont valables pour la vaste majorité des cas de traumatisme crânien au Canada.

\section{Conclusion}

On assiste depuis dix ans à une hausse significative de l'incidence des traumatismes crâniens ${ }^{2}$. Cette hausse, attribuable à de nombreux facteurs, attire l'attention sur la manière dont on obtient des soins à la suite d'un traumatisme crânien. Notre observation concernant le changement de lieu pour l'obtention de soins choisi par les personnes ayant déclaré un traumatisme crânien est importante, notamment car elle révèle une diminution des visites aux services des urgences chez les jeunes dans les dernières années. Il serait donc bénéfique, face à ce changement, d'améliorer la capacité des professionnels concernés à reconnaître les traumatismes crâniens.

\section{Conflits d'intérêts}

Les auteurs déclarent n'avoir aucun conflit d'intérêts.

\section{Contributions des auteurs et avis}

Tous les auteurs ont lu et approuvé le contenu du présent article. DPR a participé à l'analyse et à l'interprétation des données, ainsi qu'à la préparation du manuscrit. SM a participé à l'interprétation des données et à la préparation du manuscrit. GJ et WT ont participé à la préparation du manuscrit.

Les auteurs assument la responsabilité du contenu de l'article et des points de vue qui y sont exprimés. Ceux-ci ne reflètent pas nécessairement ceux du gouvernement du Canada.

\section{Références}

1. MacLeod L. Bill 149: Rowan's Law Advisory Committee Act, 2016. Legislative Assembly of Ontario. 2016; Chapter Number: S.O. 2016 C.11.

2. Rao DP, McFaull S, Thompson W, et al. Trends in self-reported traumatic brain injury among Canadians, 20052014: a repeated cross-sectional analysis. CMAJ Open. 2017:5(2):E301-E307. doi: 10.9778/cmajo.20160115.

3. Feinstein A, Rapoport M. Traumatisme cérébral bénin : une épidémie silencieuse. Revue canadienne de santé publique. 2000;91(5):326, 332 .

4. Tator CH. Concussions and their consequences: current diagnosis, management and prevention. CMAJ. 2013;185: 975-979. doi: 10.1503/cmaj.120039.

5. Zemek R, Barrowman N, Freedman $\mathrm{SB}$, et al. Pediatric Emergency Research Canada (PERC) Concussion Team. Clinical Risk Score for Persistent Postconcussion Symptoms Among Children With Acute Concussion in the ED. JAMA. 2016; 315:1014-1025.

6. Bey T, Ostick B. Second impact syndrome. West J Emerg Med. 2009; 10:6-10.

7. McCrory P, Meeuwisse W, Aubry M, et al. Consensus statement on concussion in sport--the 4th International Conference on Concussion in Sport held in Zurich, November 2012. Clin J Sport Med. 2013;23:89-117. doi: 10.1136 /bjsports-2013-092313.

8. Guskiewicz KM, Broglio SP. Sportrelated concussion: on-field and sideline assessment. Phys Med Rehabil Clin N Am. 2011;22:603-617, vii.

9. McCrory P, Meeuwisse W, Dvorak J, et al. Consensus statement on concussion in sport-the 5th international conference on concussion in sport held in Berlin, October 2016. Br J Sports Med. 2017;51(11):838-847. doi: 10.1136/bjsports-2017-097699.
10. Parachute. Lignes directrices canadiennes sur les commotions cérébrales dans le sport. 2017. En ligne à : http://www.parachutecanada.org /sujets-blessures/article/lignes -directrices-canadiennes-sur-les -commotions-cerebrales-dans-le-sport

11. Taylor AM, Nigrovic LE, Saillant ML, et al. Trends in Ambulatory Care for Children with Concussion and Minor Head Injury from Eastern Massachusetts between 2007 and 2013. J Pediatr. 2015;167:738-744. doi: 10.1016/j.jpeds .2015 .05 .036 .

12. Zemek RL, Grool AM, Duque DR, et al. Annual and seasonal trends in ambulatory visits for pediatric concussion in Ontario between 2003 and 2013. J Pediatr. 2017;181:222-228.e2. doi: 10.1016/j.jpeds.2016.10.067.

13. Beland Y. Canadian community health survey-methodological overview. Health Rep. 2002;13:9-14.

14. Faul M, Xu L, Wald MM, et al. Traumatic Brain Injury in the United States: Emergency Department Visits, Hospitalizations and Deaths 20022006. Atlanta (GA): Centers for Disease Control and Prevention, National Center for Injury Prevention and Control; 2010. En ligne à : https:// www.cdc.gov/traumaticbraininjury /pdf/blue_book.pdf

15. Arbogast KB, Curry AE, Pfeiffer MR, et al. Point of Health Care Entry for Youth With Concussion Within a Large Pediatric Care Network. JAMA Pediatr. 2016; 5;170(7):e160294. doi: 10.1001/jamapediatrics.2016.0294.

16. Angus Reid Institute: Public Interest Research. One-in-five Canadians say they've suffered a concussion playing sports; most before turning 18. En ligne à : http://angusreid.org/concussion -in-sport/

17. Lincoln AE, Caswell SV, Almquist JL, et al. Trends in concussion incidence in high school sports: a prospective 11-year study. Am J Sports Med. 2011; 39(5):958-963. doi: 10.1177/03635465 10392326. 
18. Parachute. Consignes relatives au retour au jeu suite à une commotion cérébrale. En ligne à : https://www.coach .ca/files/returntoplayguidelinesFR .pdf

19. Cancelliere C, Hincapie CA, Keightley $\mathrm{M}$, et al. Systematic review of prognosis and return to play after sport concussion: results of the International Collaboration on Mild Traumatic Brain Injury Prognosis. Arch Phys Med Rehabil. 2014;95(Suppl 3):S210-229. doi: 10.1016/j.apmr.2013.06.035.

20. Gabbe BJ, Finch CF, Bennell KL, et al. How valid is a self reported 12 month sports injury history? Br J Sports Med. 2003;37:545-547. doi: 10.1136/bjsm.37 .6 .545 . 